\title{
The Javelin Integrated Flight Simulation
}

\author{
Charles Bates ${ }^{1}$, Jeff Lucas $^{2}$, Joe Robinson ${ }^{3}$ \\ ${ }^{1}$ US Army AMCOM, AMSAM-RD-SS-SD, Redstone Arsenal, AL 35898-5000 \\ Charlie.bates@rdec.redstone.army.mil \\ ${ }^{2}$ Computer Sciences Corporation, 4090 South Memorial Pkwy, Huntsville, AL 35815 \\ Jeff.lucas@rdec.redstone.army.mil \\ ${ }^{3}$ CAS, Inc., 555 Sparkman Drive, Huntsville, AL 35814 \\ Joe.robinson@rdec.redstone.army.mil
}

\begin{abstract}
The cornerstone of the all-digital simulation for the Javelin missile system is its accurately rendered high fidelity $8-12 \mu \mathrm{m}$ infrared imagery of targets, clutter, and countermeasure effects. The Javelin missile system is a medium range, manportable, shoulder-launched, fire-and-forget, anti-armor weapon system. Javelin has two major components: a reusable Command Launch Unit (CLU) and a missile sealed in a disposable launch tube assembly. The CLU incorporates an integrated day/night sight and provides target engagement capability in adverse weather and countermeasure environments. The Javelin missile incorporates an imaging infrared seeker with a fire-andforget tracker that allows the gunner to fire the missile and immediately take cover. The on-board tracker guides the missile to the target until impact. The tracker and its ability to maintain lock on the target throughout flight emerged as the most critical aspect of the Javelin system. A cost-effective way to develop and test new tracker algorithms became a necessity. Additionally, an innovative way to determine system performance of a fire and forget imaging infrared system was needed. From these requirements came the development of the Javelin Integrated Flight Simulation (IFS). The Javelin IFS is a high fidelity all-digital simulation whose primary functions are tracker algorithm development, flight test predictions and reconstructions, and system performance assessment. The IFS contains six models: environment model, seeker model, tracker model, autopilot, six degrees-of-freedom (6-DOF) model, and gunner model. The most critical aspect of the IFS is feeding the tracker with as realistic imagery as possible. The seeker model working in conjunction with the environment model gives the IFS highly realistic and fully verified, validated, and accredited imagery of targets, terrain, and countermeasures.
\end{abstract}

\section{Introduction}

The Javelin Project Office undertook a comprehensive program for managing key system, subsystem, and component-level models and simulations, related databases, and test data. These models and simulations evolved from initial all-digital simulations supporting requirements analysis, and they have continued to support the program throughout its development life cycle. The primary simulation developed to 
represent the Javelin system was the Javelin Integrated Flight Simulation (Javelin IFS).

The Javelin IFS is a high-fidelity, all-digital simulation whose primary functions are tracker algorithm development, flight test predictions and reconstructions, and system-performance assessment. The Javelin IFS contains an environment model, a seeker model, a tracker model, a guidance model, a six degrees-of-freedom (6-DOF) model, and a gunner model. All of which are controlled by an executive and data from each model is passed via shared memory segments. The term Integrated Flight Simulation was coined to signify the use of Mission Critical Code Resources (i.e. Flight Software) as part of the simulation. This integration has proven to be the bedrock on which Javelin program successes have been built.

The Javelin's prime contractor and the U.S. Army's Aviation and Missile Research, Development and Engineering Center (AMRDEC) developed the Javelin IFS jointly during engineering and manufacturing development (EMD). Verification, validation, and accreditation (VV\&A) of the Javelin IFS was performed by AMRDEC, and accreditation by the Army Materiel Systems Analysis Activity (AMSAA). The IFS was then used to assess the performance of the system through many thousands of monte-carlo simulation runs with various environmental and attack scenarios. The data derived from this performance assessment was used to support the Low-Rate Initial Production (LRIP) phase of the program.

\section{The Javelin Integrated Flight Simulation}

The IFS represents the first simulation from AMCOM AMRDEC of an imaging fireand-forget missile system that included large sections of the Mission Critical Code Resource (MCCR) as a part of the simulation. The architecture of the IFS was unique at its creation (early 90's) in that it treated models or groups of models as independent applications that communicated through message queues and shared memory segments. This approach was driven primarily by the state of the art (at the time) of compilers, operating systems, and hardware. Initially, the Javelin system was modeled with software that was written in Fortran, ADA, C, and Pascal. Grouping each of these models into a monolithic simulation and interfacing the disparate programming languages became difficult in both implementation and maintenance.

A decision was made to create smaller components and interface them using interprocess communication features of the Unix operating system. Socket based communication could have been chosen but the overhead compared to shared memory access was significant enough to decide otherwise. This architecture, illustrated in Figure 1, is working well for the Javelin project but it isn't essential to simulating this system. There are many solutions to this problem and all would be valid. The important aspect of the IFS is the segmenting of the problem into smaller sets. Maintenance of the simulation became more manageable. 


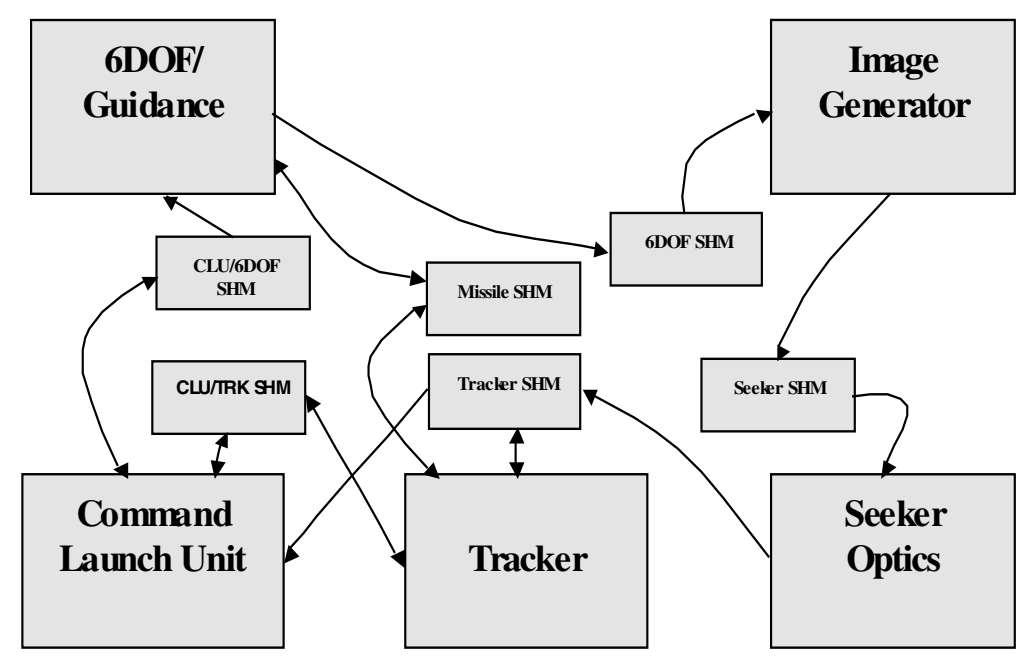

Fig. 1. IFS Block Diagram.

The IFS consists of five applications to provide modeling and interfaces for the 6DOF hardware simulation, guidance, tracking, seeker optics, environment, and gunner/launch unit modeling. An executive application coordinates the creation and removal of the message queues and shared memory segments, as well as the actual execution of the simulation applications. Each simulation application signals the other applications through message queues when another execution frame is needed.

As an imaging simulation, the IFS has struggled with the tradeoffs associated with simulation of an infrared scene. These tradeoffs include providing enough fidelity to adequately test the tracker software, the cost associated with collecting signatures that represents various objects, backgrounds, and phenomenon under a variety of conditions, and execution of enough simulation runs to provide valid statistical datasets for evaluation. These tradeoffs have resulted in developing over 25 square miles of terrain databases at $1 \mathrm{ft}$ resolution with multiple signatures as well as over 50 target models with as many as 20000 polygons each and executing in excess of 200000 simulation runs.

\subsection{The Environment Model}

The Environment Model is in short the IFS scene generator. Its purpose is to create a high-resolution image for a specified scenario from the viewpoint of the missile 
seeker and then transfer this image to the seeker optics model. The Javelin scene generator provides the user the capability to create "environments" through a scenario description file. This scenario description allows the user to specify the use of a specific section of terrain elevation and signature, objects such as tanks, trees, bushes, events such as moving or articulated parts of objects and various phenomenon such as smoke and dust clouds or fires and flares. The user may also specify atmospheric attenuation, ambient temperature, and sky temperature. At the point the missile impacts the target, the environment model calculates the intersection point in target coordinates for use in vulnerability assessments.

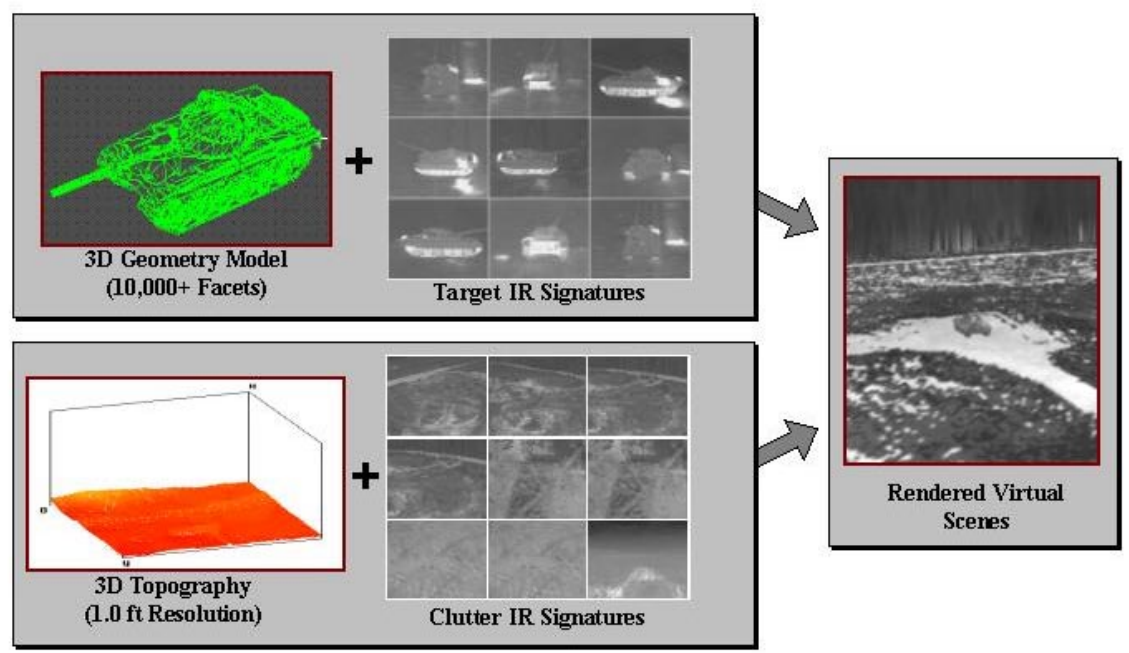

Fig. 2. Building a Scene.

From its inception, The environment model was developed for the Silicon Graphics platform. Originally, SGI provided the best platform for the creation of this type of imaging simulation as well as providing the cost/benefit ratio to afford to execute thousands of runs that might take as long as an hour each. As time and technology has progressed, the scene generator has been improved to take advantage of new features but the pace of adoption has been slow due to configuration management issues. Army acquisition rules require simulations to be accredited by an agency independent of the developers. This insures a good product but also necessarily, limits changes to the product to keep reaccredidation costs low. For this reason the Javelin target models are still rendered using shaded polygons and the terrain while texture still relies on a regularly gridded terrain skin. When the design for this software was completed, the Reality Engine series of graphics board was the best available but unfortunately 4 MBs of texture RAM was not enough for both target and terrain.

As the cost/benefit ratio becomes unbalanced due to technology shifts, the effort to modify the scene generation becomes justified and that is the case now. New thrusts 
will include converting targets and terrains to textured models in a commercially available format (i.e. OpenFlight ${ }^{\circledR}$ ).

The targets and terrain databases used by the IFS are predominately characterized as empirical datasets. Early in the program, a decision was made to not depend on predictive signatures for either target or terrain but rather gather as much measured data as possible. Initial studies of the Javelin missile system indicated that the hit point of the missile on the target would be controlled overwhelmingly by the tracker. Now that seems an obvious statement but to clarify, when all other error sources of the missile are combined for Javelin, the system can still consistently hit a point on a target smaller than its diameter. Therefore, the controlling factor is the tracker error. Of course, the tracker error is controlled by the scene itself hence the use of measured signatures.

The process by which these models were created was laborious at first but as time passed and the process was refined, automated tools lowered the workload.

The process of building target models included:

1. Obtain accurate drawings or measurements of the target.

2. Obtain calibrated infrared signatures from the target.

3. Construct a polygonal model of the target using drawings and add additional vertices to capture thermal detail as necessary.

4. Render an image of the resulting model and compare back to the measured signature.

This process seems straightforward and it is but it does result in target models that can exceed 20,000 facets.

Terrain modeling follows a similar process but on a larger scale.

1. Gather topographic information in the form of elevation data and class descriptions (i.e. vegetation types and location). This was obtained very often through survey overflights.

2. Collect calibrated signature data from different class types.

3. Create a topographical description file for the elevation data.

4. Create a signature description from the class maps.

5. Render and compare the result with the original signature measurements.

This process required a great deal of infrastructure support but the Army has capitalized on its investment by making the datasets available to other programs. This community sharing has resulted in a variety of models that provide excellent evaluation and development capabilities to the imaging tracker community.

Everyone would like to have the ability to predict signatures and it will happen at some point in the near future. However, due to VV\&A concerns at the time, the method of collecting the exact imagery for that point in time emerged as the preferred approach. No one could argue the validity of real target signatures and real terrain 
signatures. It is very likely that the datasets collected on this program will provide the means to validate predictive techniques in the future when they are available.

\subsection{The Seeker Model}

The seeker model provides an optics and signal-processing model of the Javelin system. The features include modeling for focus, focal plane array (FPA) nonlinearity, bad pixels, gain and level processing, and electronic noise. The seeker application obtains a high-resolution scene image from the environment model and creates a low-resolution image by first applying an optical blurring function. This optical blurring function varies as a function of range to a surface and provides the blurring that occurs as the missile approaches the target. The parameters of the blurring function can be varied statistically to cover the manufacturing error of the optics. After the optically processed low-resolution image is created then the effects of FPA non-linearity, bad pixels and gain and level processing are added. This resulting image represents the image that would be presented to the Javelin tracker in the real hardware. The seeker application makes this image available through shared memory to the tracker model.

\subsection{Tracker Model}

The tracker model is how the Integrated Flight Simulation got its name. The original concept was to "integrate" the real tracker MCCR code (Ada) into the IFS. The same source code base for the Javelin tracker is compiled for the missile and compiled to run on SGI processors for the simulation. This means that the same program flow is occurring on both processors. The only sections of the MCCR code not included in the IFS are the built-in-test (BIT) and the array processor functions that run on specialized chip sets in real-time language (RTL). Because the core of the tracker is preserved in the IFS, it can be said that the tracker is not simulated.

Math operations, particularly floating point calculations in the array processor sometimes differ slightly. A matching process is rigorously undertaken for each tracker delivery to identify any of these types of differences and provide compensation for them in a simulation specific portion of the tracker code. This addresses such issues as rounding differences and truncation differences.

The tracker model receives seeker imagery from the seeker model through a shared memory interface. The tracker then performs its tracking algorithms in order to compute the desired aimpoint on the intended target. From the computed aimpoint, the track errors are calculated and then sent to the autopilot where the guidance electronics command the control actuator subsystem (CAS) to move the missile.

\subsection{The Missile Hardware and Guidance Model}

The 6DOF missile application models all the hardware of the missile. This hardware includes the seeker gimbal dynamics, flight computer process timing, launch and 
flight rocket motors, the fin actuator dynamics, and the missile aerodynamics. This application also models the earth, atmosphere, and wind. The user also has the capability of simulating hardware failures to support testing of range safety algorithms in the flight software. The guidance software is included in this application rather than separating as the tracker in order to support legacy studies. The 6DOF application can run in a standalone mode and provides monte-carlo simulation capability particularly for cases where the tracker is not of concern. When used in the IFS, this application provides the missile viewpoint to the environment model to render a scene for the seeker model. It accepts the tracker error from the tracker model to provide a closed loop simulation. At initialization, the 6DOF application interfaces with the Gunner Model to position the missile and to respond to flight software mode changes as directed by the gunner model.

\subsection{The Gunner Model}

The gunner model portion of the IFS simulates the interface between the gunner and the missile hardware.

The Command Launch Unit (CLU) is the Javelin sight. It allows the gunner to find the target on the battlefield, lock-on to the target, and fire the missile. The gunner identifies and centers the target in the CLU sight then switches to the missile seeker to size the track gates around the target. When the tracker has locked on, the gunner can fire the missile.

In the IFS, the gunner/CLU model performs this same function. The scene generator knows which pixels within the seeker image are target and which are background. This target truth is available through a shared memory interface to the gunner model via an image color-coded to identify target location. The gunner model uses this image to center and size the track gates roughly around the target. Moving the target within the image is accomplished by giving the scene generator a new pitch/yaw orientation. This mimics the gunner moving the CLU/missile to position the target near the center of the image. Sizing the track gates is accomplished by setting and clearing bits in the CLU/tracker interface. Since the actual Javelin Tracker is running within the IFS, these single bit commands are the same as those sent from an actual CLU. Monte Carlo errors are applied to track gate size and position. These error distributions are based on measurements of gunners in the field.

\section{Next Generation IFS}

As stated earlier, when the cost/benefit ratio is unbalanced enough then upgrading is justified. The users of this simulation (Javelin PMO, AMCOM AMRDEC, and Raytheon) have determined that case exist now. With the availability of stable objectoriented language compilers (i.e. $\mathrm{C}++$ ) and new players in the high-end graphics market as well as new computing platforms, there are a myriad of opportunities to gain new efficiencies or attempt more complex problem sets. 


\subsection{New Simulation Framework}

The first item of improvement has been to adapt to a new simulation framework developed and promoted by AMCOM AMRDEC and referred to as the Common Simulation Framework (CSF). CSF was developed to aid AMRDEC in promoting a common set of tools in its development and evaluation business. CSF is an objectoriented and shared object approach to building simulations. Users are encouraged to develop models as classes with interfaces that are similar to the interfaces of the real hardware. A shared object is created with this class definition and libraries of these classes are gathered to construct a simulation. The simulation is constructed by instantiating the classes and connecting through their open interfaces. The obvious benefit is the promotion of software reuse. As different types of models are created, eventually a library of missile parts will be developed. Software reuse, however, is not the prime motivator. Missile systems are never just created and then statically maintained for the lifetime of the missile. As new threats are realized, improvements are necessary and to aid in this effort a simulation that allows itself to freely adapt new models is desired. One example would be as countermeasures are created that defeat the current tracker, tests could be perform on other vendors trackers to keep abreast of new technologies.

The Javelin IFS has been transferred to this new framework and is being tested to insure that all capabilities are maintained as well as maintaining a valid system simulation of Javelin.

\subsection{New Scene Generator}

With the explosion of new 3D graphics capabilities, vendors are releasing new products at a surprising rate. This certainly opens up the opportunity to increase capability and lower costs at the same time. For this reason, a new scene generator is being developed to take advantage of all the scene generation techniques available. The new scene generator will use models and terrain databases retuned and converted to use the OpenFlight ${ }^{\circledR}$ format and will be rendered using scene graphs. The volumetric effects such as smoke and fire will be implemented using 3D texturing and the increasing availability of texture RAM. When it is possible, some phenomenon will be implemented using particle effects. All these techniques along with efficient use of levels-of-detail will provide efficiencies necessary to implement increasingly complex testing scenarios such as shadows and reflective processes.

\section{Conclusion}

The Javelin IFS is a fully verified, validated, and accredited model of the Javelin missile system and its sub-systems. Because of the fidelity and validity of the IFS, Javelin can with confidence use the IFS to determine system performance, execute flight predictions and reconstructions, and perform tracker design and analysis. The IFS is an example of a simulation that is an integral part of a current US Army missile program and is being used on future missile programs as well. 